\title{
Biomarkers Expression in Human Seminiferous Epithelium
}

\author{
R. Sá*, C. Miranda*, C. Leite*, I. Malheiro**, F. Carvalho***, J. Silva****, A. Barros***,**** and \\ M. Sousa* \\ * Lab of Cell Biology, UMIB, Institute of Biomedical Sciences Abel Salazar, University of Porto, \\ Porto, 4099-003 \\ ** Lab of Cytogenetics, Institute of Biomedical Sciences Abel Salazar, University of Porto, Porto, \\ 4099-003 \\ *** Department of Genetics, Faculty of Medicine, University of Porto, Porto, 4200-319 \\ **** Centre for Reproductive Genetics Prof. Alberto Barros, Porto, 4100-012
}

Spermatogenesis is a complex process of cell proliferation, meiosis and differentiation $[1,2]$. In order to determine the genetic mechanisms that control this process we need to develop adequate methodologies for the purification of stage-specific germ cells. In this study we aim to evaluate four markers (c-kit, oct-4, Integrin $\alpha_{6}$ and Integrin $\beta_{1}$ ), used to isolate spermatogonial stem cells in mammals, as possible markers to isolate/sort testicular adult stem and progenitor cells in humans.

Under informed patient consent, germ cells from patients with secondary obstructive azoospermia or anejaculation submitted to treatment testicular biopsies [3] were isolated by micromanipulation [4, 5]. Lymphocytes were isolated from peripheral blood of a healthy male by Ficoll gradient centrifugation, and CD34+ cells isolated from human umbilical cord blood by using a Magnetic Activated Cell Sorter. Ribonucleic Acid (RNA) was isolated and reverse transcribed. Complementary Deoxyribonucleic Acid (cDNA) of targeted genes was amplified with PCR. Proteins were immunolocalised either using monoclonal/polyclonal primary antibodies and fluorochrome-conjugated secondary antibodies in isolated cells, either by immunohistochemistry of paraffin sections of testicular biopsies.

RT-PCR results showed the presence of all markers in every sample (diploid germ cells, primary spermatocytes and lymphocytes), except for c-kit, which was not present in primary spermatocytes (Figure 1). Oct-4 expression was observed in spermatogonia by immunocytochemistry means, while for c-kit there were two kinds of spermatogonia population, positive and negative (Table I, Figure 2). After this stage, all markers were positive albeit at different intensities. There were two populations of spermatogonia positive and negative for both Integrins. Spermatocytes were positive for both markers and during spermiogenesis their expression disappeared. Cells from the haematopoietic system and Sertoli cells also expressed all four markers (Table I).

The results suggest that these markers used to isolate bovine and rodents' spermatogonia are not applicable to humans, once c-kit and Oct- 4 were found in other germ cells beside spermatogonia. $\alpha 6$ and $\beta 1$ integrins, which were though to be specific of the connection between the basal membrane and the spermatogonia were detected through almost all the germ cells. This suggests that, in humans, these molecules might be involved in Sertoli cell and germ cell connection until spermiation. Expression of all markers in lymphocytes and CD34+ indicates that the presence of these markers per se will not avoid contamination of germ cell fractions using cell sorting procedures.

\section{References}

1. Clermont, Am J Anat 112: 35-51, 1963

2. Clermont, Physiol Rev 52:198-236, 1974

3. Sousa et al., Hum Reprod 17:1800-1810, 2002

4. Sousa et al., Hum Reprod 17:161-172, 2002

5. Sá et al., Biol Reprod 79:962-975, 2008 


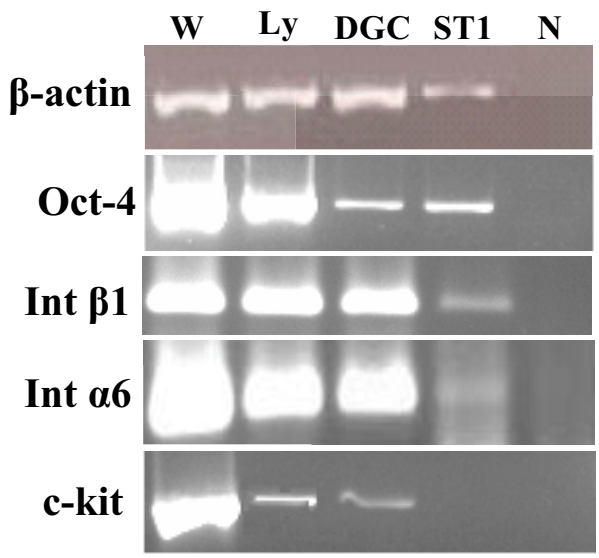

Figure 1. RT-PCR - Expression of markers of adult progenitor cells in male germinal epithelium and lymphocytes. B-actin was used as the loading control. W, whole testicular tissue; Ly, lymphocytes; DGC, diploid germ cells; ST1, primary spermatocytes, $\mathrm{N}$, negative control (DNA replaced by water).

Table I. Imunocytochemistry expression of progenitor cells markers in male germ and haematopoietic lines.

\begin{tabular}{lcccc} 
& c-kit & Oct-4 & Int $\alpha_{6}$ & Int $\beta_{1}$ \\
\hline Spermatogonia (SG) & $-/+$ & +++ & $-/+$ & $-/++$ \\
Primary spermatocytes (ST1) & + & +++ & ++ & ++ \\
Secondary spermatocytes (ST2) & + & +++ & & + \\
Spermatids (SD) & $-/+$ & $-/+++$ & + & - \\
Spermatozoa (Sz) & - & - & - & +++ \\
Sertoli cells (SC) & + & +++ & +++ & + \\
Mature lymphocytes (Ly) & + & ++ & +++ & + \\
CD34+ & ++ & ++ & +++ & + \\
\hline
\end{tabular}
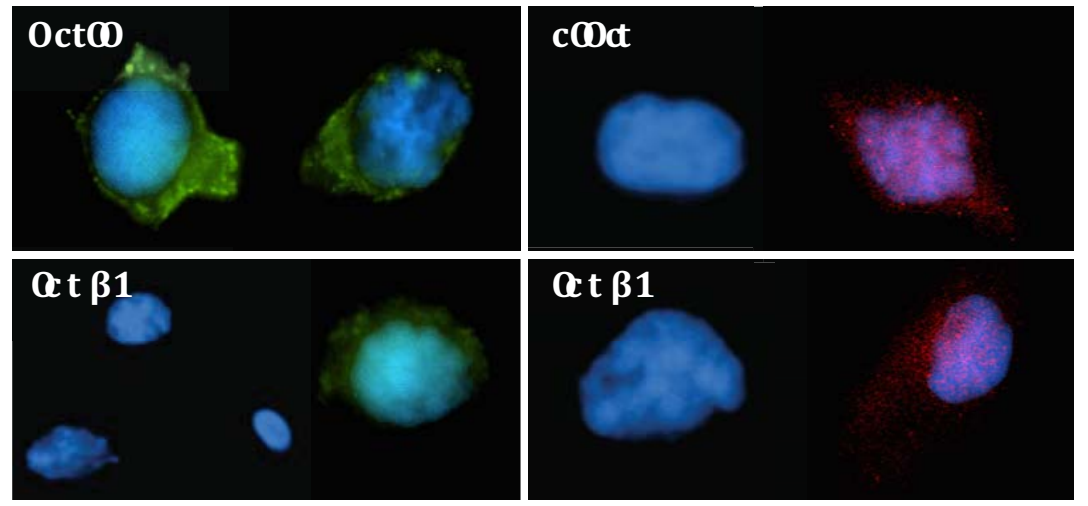

Figure 2: Imunocytochemistry revealed the existence of two populations of spermatogonia for c-kit and for both integrins. Images obtained in a Nikon Eclipse model E400 Epifluorescence Microscope. Original magnification: $100 \mathrm{x}$

Study supported by Foundation for Science and Technology, Ministry of Science, Technology, and Superior Education, with a Ph.D. grant to R.S. (SFRH/BD/23616/2005) and research grants to R. Sá and M. Sousa (UMIB). The work was presented at the $2^{\text {nd }}$ Joint Congress of the Portuguese and Spanish Microscopy Societies. 